\title{
Engineered Perception Architecture for Healthcare
}

\author{
Mihai Nadin \\ Institute for Research in Anticipatory Systems \\ Ashbel Smith University Professor \\ The University of Texas at Dallas \\ Richardson, USA \\ nadin@utdallas.edu
}

\author{
Asma Naz \\ Institute for Research in Anticipatory Systems \\ The University of Texas at Dallas \\ Richardson, USA \\ asma.naz@utdallas.edu
}

\begin{abstract}
Experiencing sensory-pleasing environments and recreating perceptions of past lived environments can benefit patients greatly in coping with irreversible conditions (e.g., dementia) or during healing after traumatic experiences. Such environments can evoke emotional responses that are associated with a patient's positive memories of a place, an activity, or people. A design concept of an intimate architectural space informed by knowledge of anticipatory systems was developed for the purpose of generating variable personalized environments. In particular, such environments are designed for patients, according to their individual demands and expectations. The design is guided by aesthetic data acquired through perceptual user studies. Such adaptive spaces can be delivered to a large number of users in healthcare in the form of Architecture-as-Service (AaS). The research presented expands the notion of AaS and is premised upon an IoT application.
\end{abstract}

\section{CCS CONCEPTS}

\section{- Human-centered computing $\rightarrow$ Interaction design;}

\section{KEYWORDS}

Adaptive, Architecture-as-Service

(AaS), interaction, perception, affective space, dementia

\section{ACM Reference format:}

Mihai Nadin and Asma Naz. 2019. Engineered Perception Architecture for Healthcare. In Proceedings of PETRA '19 conference. Rhodes, Greece. https://doi.org/10.1145/3316782.3321525

\section{INTRODUCTION}

The need for providing sensory-pleasing spatial environments in hospitals, or a "homey" environment in nursing homes has

Permission to make digital or hard copies of all or part of this work for personal or classroom use is granted without fee provided that copies are not made or distributed for profit or commercial advantage and that copies bear this notice and the full citation on the first page. Copyrights for components of this work owned by others than ACM must be honored. Abstracting with credit is permitted. To copy otherwise, or republish, to post on servers or to redistribute to lists, requires prior specific permission and/or a fee.

Request permissions from Permissions@acm.org.

PETRA '19, June 5-7, 2019, Rhodes, Greece

(C) 2019 Association for Computing Machinery.

ACM ISBN 978-1-4503-6232-0/19/06 ...\$15.00

https://doi.org/10.1145/3316782.3321525 been deemed beneficial for treatment of patients with specific medical conditions. Patients respond better to treatments when healthcare environment can stimulate the feeling of being "at home." Pleasing environments reduce anxiety, stress, pain and encourage positive attitude towards treatment. In the article "The Comforting Fictions of Dementia Care," MacFarquhar [1] discusses the significance of "memory-care units" in nursing homes that attempts to rebuild past lived environments for dementia patients. The Lantern of Chagrin Valley and the Hogeweyk (Dementia Villages) are current attempts at building upon neuroscience-based suggestions for facilities that allow patients to cope with irreversible conditions, or at providing treatment of patients who have undergone traumatic experiences. With the aid of visual, tactile or auditory props and simulation of scenes, illusions of a patient's memories are recreated in order to provide a sense of safety and comfort. Having patients reside in individual "care units" with nostalgic reconstructions of their own past environments-e.g., a façade or a porch of a house, a street, a bus stop or a beach front set up with heat lamps and ambient sound-help soothe the mental anguish they suffer from. Moreover, carrying out daily activities feels more natural, comforting and convincing when that environment reminds them of past lived spaces where they have conducted similar activities [1]. Having the ability to adjust sensory-stimulants in a hospital environment is also imperative for people or children with autistic spectrum conditions who have low tolerance or hyper-sensitivity towards specific colors, sound, lighting conditions or size [2].

In this paper, a concept of an adaptive, interactive space is proposed as a real-world patient recovery or treatment room in a hospital or a care unit in a nursing home. The "room" offers variable spaces for its patients through changing perception of its affective qualities according to each patient's context. The room has the capacity to transform itself to adapt to a patient's immediate needs, providing emotional comfort to support his/her mood or activity. The space is personalized i.e., connected to the patients' memories of past lived spaces (having positive associations) by creating sensations or imageries that would remind them of those memories. User studies have been presented to explain the process of aesthetic data collection that is imperative to initiate design. The paper further discusses implementation of this concept within the 
framework of Internet-of-Things (IoT) in order to provide a data- driven spatial solution as a design service and produce personalized spatial environments on demand to a larger population. The premise for this is our concept of Architectureas-Service $(\mathrm{AaS})$ for which a model based on an application of blockchain technology was developed [3]. In addition, we build upon the knowledge specific to the anticipatory aspects of spectrum diseases (Alzheimers, Parkinsons, autism) developed by Nadin $[4,5,6,7]$. We suggest, with our solution, an adaptive space in which long-term memory is enlisted as a factor conducive to influencing behavior.

\section{RELATED TOPICS}

In this section, we review the current practices of interactive multisensory spatial environments in healthcare institutions for patient's health and wellbeing. The concept of affective space creation in traditional architecture is discussed with an overview of the psychophysiological and cultural associations of various sensory design parameters of architectural spaces.

\subsection{Interactive Environments in Healthcare}

Ambient intelligence enables "smart" patient rooms to monitor patient's health through embedded sensor networks, wearable devices, motion and gesture recognition systems, data memories, as well as environmental monitoring systems for temperature, humidity or lighting conditions [8]. Patients can also control their own surroundings-e.g., bed, TV, window and/or lighting conditions - to an extent with use of touchpad devices, displays or graphical user interfaces [9].

Beyond these ambient intelligent-based systems, interactive technologies have been explored in building immersive multisensory environments for children and patients with psychological or physical disabilities to encourage active physical and cognitive engagement with surrounding. Studies have shown that interactive multisensory environmentscreated with props, imageries, video projections, color, light or image changing LEDs-help enhance patient experience, reduce anxiety, pain or distress, stimulate curiosity and increase motivation towards treatment [9]. In the absence of natural views inside the hospital wards and examination rooms, ambient environments (images, video, sound, lighting conditions, aromatherapy etc.) provide a positive distraction for patients. Theme-based environmental designs in patient or examination rooms (for instance, the MRI room) have also contributed in drastically reducing the need to calm patients through sedation before treatment [10].

As emotional wellbeing is crucial for better response to treatment, many hospitals turn their waiting rooms and corridors into therapeutic playgrounds for children and adolescents in order to reduce their feelings of insecurity, uncertainty, anxiety, pain or distress associated with medical treatments and being away from home. "Woodland Wiggle" is an interactive wall (a TV screen) in the Royal London Children's
Hospital that captures patients' motions through sensors and visually place them inside the digital environment with animated characters, animals, nature, rainbow and various other effects [11]. In the Great Ormond street hospital in London, "The Nature Trail" is an LED screen-based interactive project that turns a corridor into an abstract "digital forest" with imageries of forest animals and trees [12]. Based on the children's positions (located through sensors), the wall illuminates images of animals for them to playfully engage with.

Some interactive projects involve changing the overall spatial quality of the room by modifying wall color or ambient lighting. In Florida Hospital for Children in Orlando, patients can select and change the LED lighting-based wall color through a touch screen user interface [13]. This ability impacts the patient's mood positively as they can not only choose a desirable color but also have certain control over their surroundings. Interactive installation ROOMI is designed for cancer patients to change the colors for their rooms as a way to reduce stress and cope with their emotions [14]. Based on the mood they are in, the patients use a pillow-like interface to select a corresponding color mode that changes the wall colors (through ambient LED lighting) in real time.

Sensor-based projection techniques are used in some hospitals to create immersive environments for sensory stimulation. Interactive displays are projected on walls or floors through ceiling or wall mounted projectors. Virtual reality gesture-controlled technology and 3D depth-tracking software sense the patients' motion, hand movement or finger gestures and enable them to engage with the colorful graphic displays in real time [15].

\subsection{Meaningful Affective Space Design}

Architectural space perception is a complete sensory experience that is visual, auditory, olfactory and tactile. In the perceptual process of the quality of a space, one perceives not only the individual properties of light, color, texture, material, but also the size, scale, proportion, openness, enclosure and the interrelationships between these elements [16].

A perceived space has affective dimensions that elicit different emotional responses from an observer. A space may feel warm, cool, spacious, intimate, exciting, calm, soft, cozy, dark, bright, open, scary, enclosed, narrow, pleasant, beautiful, unpleasant, inspiring, informal, formal, relaxing, depressing, natural, artificial and so on. These are psychophysiological aspects of space that influence an occupant's mood, emotional state, functions, attitude, choices and behavior [17].

Perception of space is highly subjective, contextual and relative to current and previous experiences. It is not only the basic sensations of sensory design elements (color, light, materiality etc.), but also one's subjective context-i.e., cultural associations, memories, experiences, aesthetic preferences, previous and prevailing emotional states - that play a part in how a space is perceived. Perception and mood are also interdependent, one impacting the other [18]. 
In his book Poetics of Space, Bachelard [19] states that our perception of comfort or discomfort, safety or danger, warmth or coolness, spaciousness or intimacy, openness or enclosure is derived from the architectural imageries and sensations we reconstruct from our memories and imagination of past lived spaces. These architectural imageries may be formed from the openness, enclosure, darkness, light or warmth of an attic, a living room, a passageway or a fireplace of past domestic habitats. Both in The Lantern of Chagrin Valley (USA) and at Hogeweyk (Holland), these observations are incorporated in the facilities.

Architectural space design is concerned with meaning. The lighting condition, openings, size and shape of a room has significant impact on how the occupant will feel and behave in that space [17]. The sensory design elements of color, light, texture and material have strong psychological, symbolic, metaphysical and cultural meanings which the architect explores in an attempt to evoke meaningful emotions and enhance aesthetic experiences for the occupant [20]. A space becomes meaningful when it successfully conveys its function to its occupant. For instance, the colors, materials, lighting condition and amount of opening or enclosure of a bedroom should evoke feelings of calmness, quietness and comfort for the occupant to sleep or take rest.

2.2.1. Perceived Affective Qualities of Color. Color, as a psychophysiological stimulant, has strong impact on human emotions and behavior. In the color spectrum, red, orange, yellow and their combinations are known as warm colors, while blue, green, purple and their combinations are known as cool colors. The concept of warm and cool colors are sensorial emotions related to perception of colors. Studies and observations have led to certain affirmations about colors impacting human psyche and depth perception. Warm colors feel more exciting, while cool colors feel more calming [21]. Warm colors also appear closer than cool colors [22]. Colors have strong natural, biological as well as cultural connotations [23]. Red is a symbol of romance, power, anger, violence or passion. In some cultures, pink is recognized as a feminine color. A study conducted in a male prison demonstrated that male aggression among inmates reduced drastically after the interior walls were painted pink [23].

2.2.2. Perceived Affective Qualities of Other Sensory Elements. Interactions of color, light, texture and material have impact on perception. Certain observations affirmed that warm and darker colors can make a space appear smaller than cool and brighter colors [21]. Colored illuminations have impact on bodily sensations and perception of space [24]. In a study conducted on multi-sensory perceptual process of architectural spaces related to colored illuminations, some common patterns could be observed. For example, blue illuminations appeared to be "cold" and "floating" to most participants and instilled feelings of discomfort and loneliness when its size appeared too large [24]. Darkness or brightness of a color impact the perceived visual weight, softness or hardness, warmth or coolness of a material [25]. Texture-a surface property of a material-has perceived affective qualities that derives from the material's age, structure, robustness or permanence. A stone with a rough texture may feel more exciting and "honest" while plaster may appear comparatively dull [25].

2.2.3. Space Perception and Sound. Auditory information contributes in perception of affective spaces [26]. Perception of space and sound are interrelated [27]. The sound of footsteps in a room may inform one about how large the space is and what material it is constructed of.

\section{AN ADAPTIVE INTERACTIVE ROOM}

This paper presents a design concept of an interactively modifiable architectural space that has the capacity to generate, in real time, personalized sensory environments for each occupant in order to adapt to their needs. We also try to facilitate the deployment of recognizable incentives for maintaining an active life, even within the confines of the physical space. This concept can have practical applications in the field of healthcare, as a recovery room or a patient's room in a hospital, a care unit in a nursing home or a rehabilitation center where patients can greatly benefit from having their sensory experiences customized according to their own, individual needs.

The room will have modifiable surfaces (walls, floor and ceiling) with embedded interactive computing systems (sensors and actuators). The sensory design attributes, such as light, surface color, brightness levels, sound, surface texture and material are modifiable. In the patient-room interaction, the room will sense (with use of ambient intelligence-based systems) the mood or intended activity of the patient and modify its overall spatial quality-i.e., feel and appearance-by modifying the sensory design attributes. The spatial environment of the room can be thus customized so that it is conducive to the patient's immediate needs.

The spatial modification pertains to modification its affective dimensions. The space will change its perceived level of warmth, coolness, openness, enclosure, intimacy, coziness, spaciousness, or calmness of a space. A "calm" and "soothing" spatial environment can be generated when the patient wants to take some rest or sleep. An "exciting" or "stimulating" space can be generated when the patient is feeling bored. A "bright" and "open" space can be generated when the patient feels claustrophobic. For patients suffering from dementia, the spatial environments created will aim to enhance their prevailing emotional states, generating sensory experiences to cater to their individual, subjective feelings of safety, solitude, excitement, comfort, intimacy, calmness or tranquility. See Figure 1. 

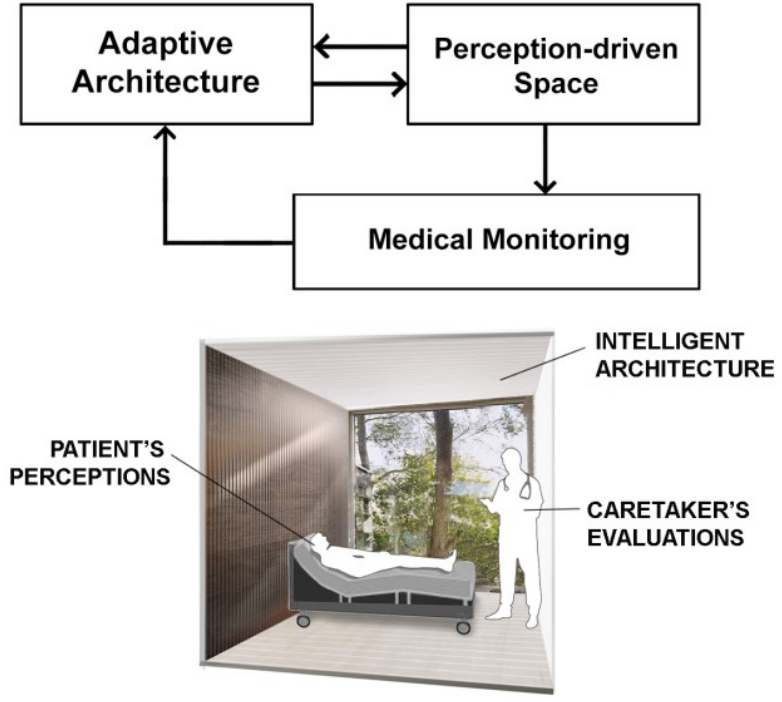

Figure 1: Structural components of AaS deployment.

\subsection{Perception-Driven Space Transformation}

Personalization of space occurs at a perceptual level without use of any props or direct physical changes to the room's size or shape. The design of interaction pertains to the understanding of the perceptual process of affective dimensions of space: i.e., how specific sensory elements can be manipulated, and spatial imageries can be created (e.g., a specific lighting condition, views or a sound environment) that can evoke desirable emotional responses from the patient. For example, one can find the meaning of safety and comfort in the bright, warm light glowing on a rustic texture of a stone wall (reminiscent of a fireplace). The sound of rain falling on the window pane may enhance the feeling of tranquility. The scent of autumn leaves in the air may make one feel vibrant. Designing affective spaces require the understanding of how these various sensations of color, light, materials or sound (and their interrelations) are perceived to derive subjective meanings of safety, security, solitude or comfort for the patient.

\subsection{Aesthetic Data Acquisition}

The design of this perception-driven adaptive space is informed by aesthetic data on correlations between perception and the sensory design elements. Aesthetic data informs the designer the extent to which one or more sensory elements (color, light, texture etc.) can be manipulated in order to create environments that evoke desirable emotions in the observer.

Some aesthetic data is needed to initiate design. Perceptual studies can be conducted to examine impact of color, light, texture, material, sound or any other architectural elements (windows, openings etc.) on various perceptions, such as feelings of comfort, discomfort, spaciousness, calmness, coziness, pleasantness, warmth etc. A set of design guidelines can be formulated from these aesthetic data to be used as a basis for human-space interaction [28]. For perceptual studies, participants must be selected from similar socio-cultural backgrounds in order to find generalized observations, affirmations, patterns or trends. Patients' medical data can also contribute in guiding design (as part of aesthetic data) concerned with their personal choices and desirable environments necessary for their health and wellbeing.

\subsection{Design Data for Sensory Design Elements}

This section demonstrates a perceptual user study that was conducted to collect aesthetic data related to specific sensory design elements. The study examined impact of color, brightness, texture and material on perceptions of warmth, coolness, spaciousness, intimacy, excitement, calmness and overall comfort of a space.

The user study was conducted in a six-sided immersive CAVE-type virtual reality (VR) system. A total of 32 participants were selected from a university campus, comprising mostly of undergraduate, graduate and post-graduate students. The age range of majority of the participants $(91 \%)$ was between 18 40 (mean $=27, \mathrm{SD}=4.8)$. A set of virtual living spaces were presented to the participants. These spaces were simulated with two types of colors (orange and blue), brightness levels (dark and light) and texture graininess (rough and smooth). The study asked participants to rate these spaces in terms of the examined affective dimensions of space. Both quantitative and qualitative studies were performed and analyzed. Details of the user study (simulation and study methods, data analysis, results and validation process) were presented at a Virtual Reality Conference and later published [29].

3.3.1. Quantitative Data Analysis. A mixed-design factorial ANOVA was performed with three repeated-measures factors and one between-subjects factor. Data analysis was conducted with IBM SPSS 24. Data was considered statistically significant at $\mathrm{p}<0.050$.

The study found statistically significant main effects of color and brightness (and their interactions) on perception [29]. Figure 2 demonstrates the main effects of color. Color was found to have significant impact on perceptions of warmth, coolness, excitement, calmness, intimacy, comfort, as well as environments for working and resting. The color orange was found significantly warmer $\left(F(1,30)=153.162, \eta^{2}=.836\right)$, more exciting $\left(\mathrm{F}(1,30)=22.087, \eta^{2}=.424\right)$ and more comfortable $\left(F(1,30)=31.772, \eta^{2}=.514\right)$ than the color blue at $p<.001$ for all measures. Orange was found significantly more intimate $\left(F(1,30)=4.960, p<.050, \eta^{2}=.142\right)$, and more preferable for resting $\left(\mathrm{F}(1,30)=10.732, \eta^{2}=.263, \mathrm{p}<.005\right)$ and working $\left(F(1,30)=10.247, \eta^{2}=.255, p<.005\right)$ than blue. Blue was found significantly cooler $\left(F(1,30)=97.117, p<.001, \eta^{2}=.764\right)$, and $\operatorname{calmer}\left(\mathrm{F}(1,30)=4.918, \mathrm{p}<.050, \eta^{2}=.141\right)$ than orange. 


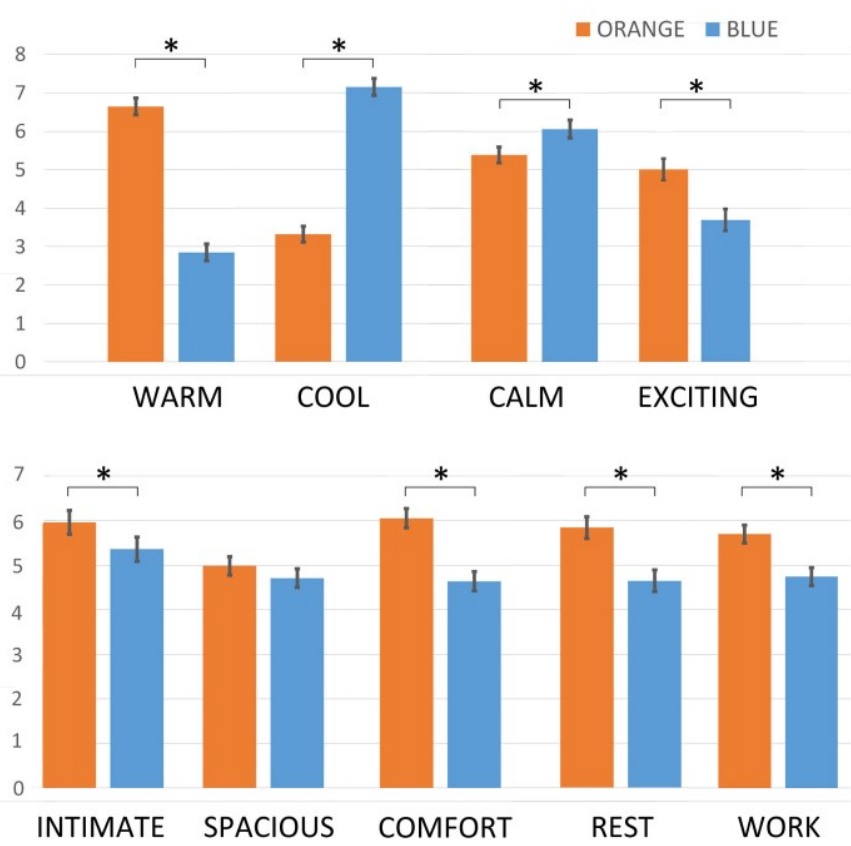

Figure 2: Main effects of color. Error bars represent standard errors.

3.3.2. Qualitative Data Analysis. A qualitative study with openended questions was conducted to ask participants about their desired spatial qualities in order to establish correlations between the spatial characteristics and perception [30]. In a "pseudo" interaction, the participants were asked to verbally describe the modifications they would prefer in order to achieve specific spatial characteristics. Their responses were categorized into themes or categories for analysis. Aesthetic data was gathered through inferences drawn from their suggestions.

The results of both quantitative and qualitative studies could be combined to draw a set of correlations between design parameters and perception as follows. These aesthetic data can be used to manipulate color, brightness, texture and material to create desirable impact on perception.
1. Color was found to have the most significant impact on perceived level of warmth, coolness, excitement, calmness, spaciousness and comfort, as well as the perceived comfort for resting and working. Warmer colors felt more exciting, warm and intimate than cooler colors.

2. Brightness had a significant impact on perceived level of spaciousness of a space. Brighter spaces felt more spacious.

3. It could be inferred that visual weight of materials influenced perceived level of spaciousness. Visually lighter materials (fabric, soft wood etc.) enhanced the feeling of spaciousness.

4. Correlations could be inferred between texture and perceived level of excitement or calmness. Smoother texture increased perceived level of calmness.

5. The perceived "softness" of a space could be associated with both color and visual weight of materials used on the surface. Muted colors and visually lighter materials enhanced the feeling of "softness" of a space.

\subsection{Design Data for a Virtual Window}

Personalization of spatial experience can also occur through various other architectural elements that impact perception. For instance, a window (or any opening) has significant impact on perception of specific affective dimensions of a living space as well as the emotional states of occupants. The size, shape and location of a window and the views it affords can be meaningful to a patient's mental state and wellbeing in a room. A window with a pleasant view-as a connection to the external worldmay influence perceived feelings of spaciousness, openness, cheerfulness and overall comfort of the space [30].

An online user study was conducted to find out the correlations between attributes of a window and occupant's perceived level of comfort [3]. The participants consisted of a total of 97 students and professionals with a background in art, design and architecture. $30.9 \%$ of the participants were in the age range of $18-25$ and $48.4 \%$ belonged in the age range of $30-$ 45 . The participants were presented with computer generated images of the interior of a room containing a single window.

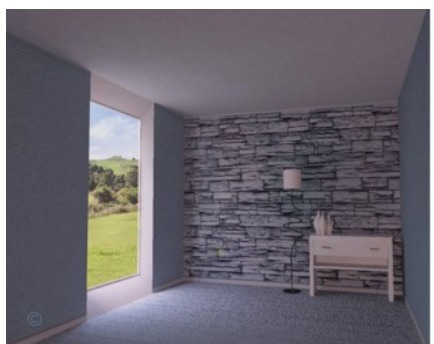

(a)

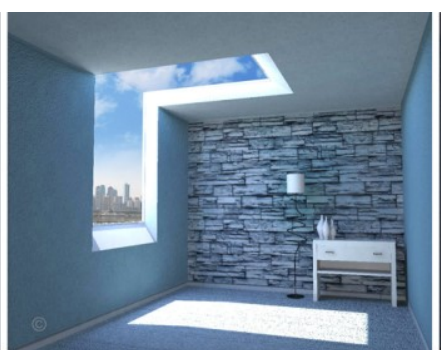

(b)

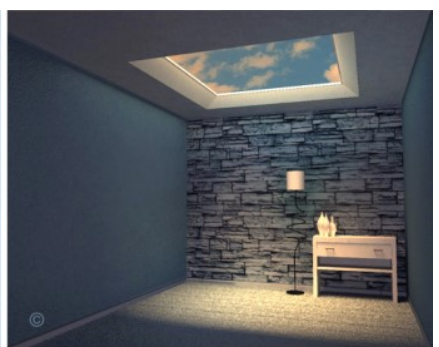

(c)

Figure 3: (a) Window shape = elongated rectangle, location = wall, view = green expanse, illumination = early morning. (b) Window shape = L-shape, location= ceiling and wall, view = city, illumination = midday. (c) Window shape = square, location = ceiling, view $=$ sky, illumination $=$ sunset. 

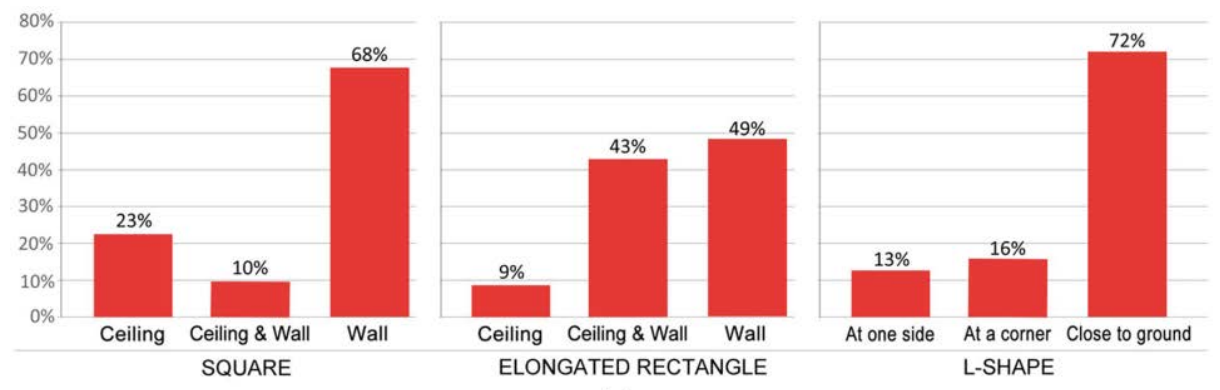

(a)

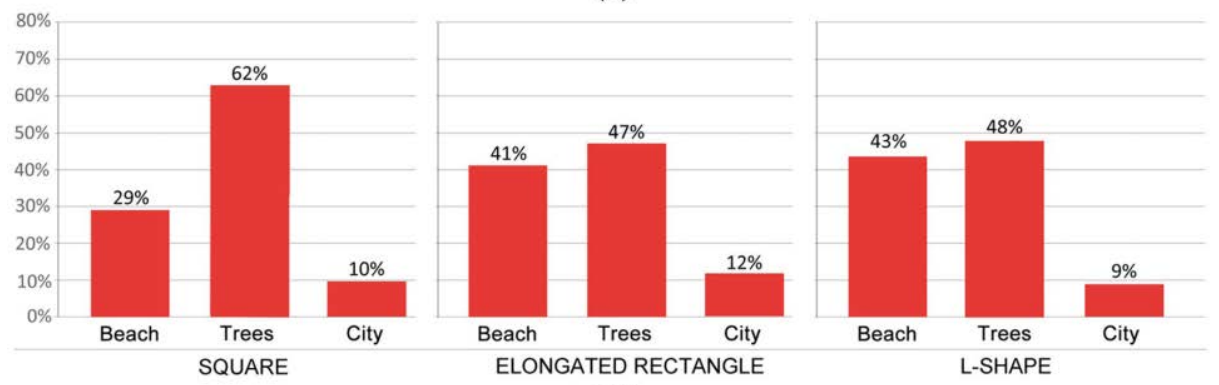

(b)

Figure 4: (a) Preferences of location for each shape. (b) Preferences of exterior views for each shape located on the wall.

The study presented the following choices for the window:

a. Three different window shapes: square, elongated rectangle and L-shape.

b. Three different locations: on the ceiling, on the wall and combined (ceiling and wall).

a. Three exterior views: a large-scale ocean or sky, a mediumscale green expanse, and a small-scale street or city view.

b. Three natural lighting conditions or illuminations: early morning, midday, and sunset.

Figure 3 demonstrates images from the study. Participants were asked to select their desired shape, location, exterior view and natural lighting condition for the virtual window. Data analysis revealed that most participants preferred the elongated rectangular shaped window to be located on the wall (Figure 4-a). Most participants preferred the view of a green expanse and an early morning illumination with softer shadows (Figure 4-b).

\section{AN IOT-BASED SPATIAL SOLUTION}

The idea of this data-driven interactive room can be further explored within the framework of Internet-of-Things (IoT). Internet-of-Things (IoT)-a network of computing devices, sensors, actuators and predictive technologies-is used for energy conservation and management of "smart" homes via cloud-based technology. Smart environments equipped with ambient intelligence and humanized computing manage and control a home environment through "smart" interactions [31].

In the article "Architecture as service: a case of design on demand (DoD)," a concept of the personalized data-driven space solution-constructed within the IoT framework and supported by blockchain technology-was proposed to be delivered to a large number of users as a "service" on demand, i.e., as and when needed [3]. This "adaptive" space requires the integration of intelligent and smart materials, management and manipulation of a broad range of architectural elements in order to provide perception-based variable spaces to occupants per context (i.e., personal needs). The implementation of such an idea is deemed possible only through IoT that allows large number of data processing, connectivity and unlimited computational resources on the cloud. The article further explained the necessity of blockchain technology to support the idea of a personalized AaS that must handle data processing and interactions without any centralized control, enable digital ownership and provide IP protection and trust that no other configuration can assure [3].

Facilitated by IoT, the units or rooms in a nursing home or a healthcare institution can provide this service through the use of smart devices, wireless sensors, smart and intelligent materials with transformable properties (temperature, reflection, refraction, texture, opacity etc.), sound systems, smart surfaces (walls or windows) and computing devices with wireless sensors, effectors, multimedia, LED screens, real-time video displays. Each room will provide perception-based variable environments personalized for each patient (Fig. 5) by transforming color, texture, materials, windows, views, lighting conditions, sound environments based upon personal context (medical record, taste, culture, activity etc.). For instance, a new virtual window can transform in shape or size and provide a specific view or lighting condition of choice. A moonlit sky, a beach or a street life of a city can be video streamed with sound 
in real time. Ambient noises of birds, water or wind can further enhance the spatial environment for the patient.
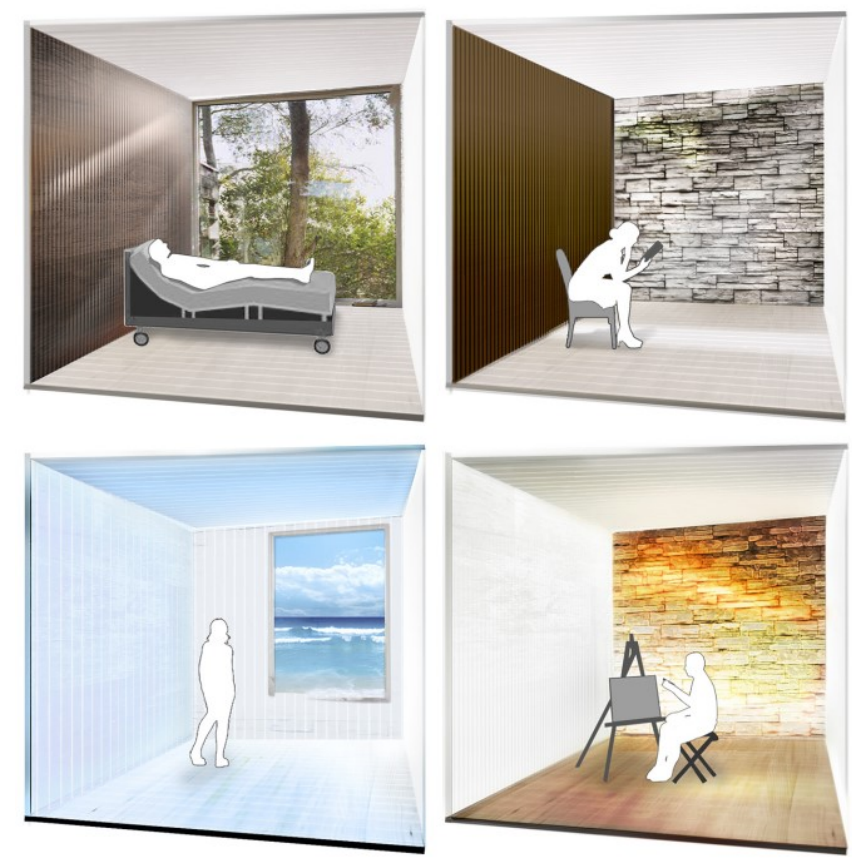

Figure 5: Surface color, texture, material, lighting conditions, windows and/or views are changed to evoke feelings of safety, security, calmness, excitement, tranquility, curiosity and so on.

Data drives the perception-based variability of space (Figure 6). Types of data also include aesthetic perceptual data, patient's demographic and socio-cultural data. Ambient intelligent devices can monitor and gather data on patient's activity [32]. Data will also be gathered through a network of sensors: such as emotional state assessment, motoric expression, gesture recognition, face recognition, health monitoring, voice recognition, haptic and activity recognition sensors. Sensors will also receive environmental data (temperature, humidity, dust etc.).

Data is the transactional element in IoT. A large amount of data is needed to be stored and retrieved when needed to create variable spaces. The cloud-based transactions of data for "smart" architectural elements and devices can be securely recorded and verified by the blockchain technology of IoT [3]. The blockchain, as a digital ledger of transactions ensures a decentralized service [33]. It will ensure validity and built-in robustness, protect intellectual property and provide secured transaction of data for both the designer (architectural service provider) and the user (patient).

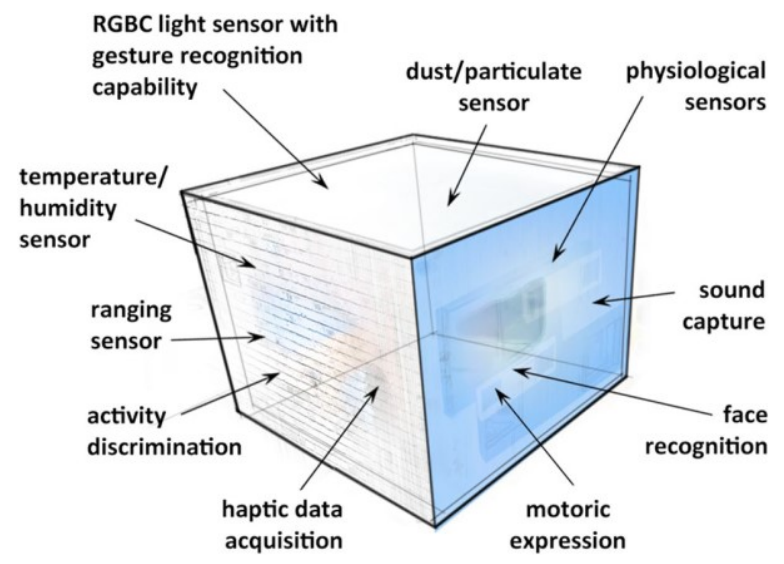

Figure 6: Control engineering: data collection and monitoring.

\section{FUTURE RESEARCH POSSIBILITIES}

There are several directions in which this space concept can develop through further research and investigation. The technological innovations in the areas of machine learning (artificial intelligence) and nanotechnology open new possibilities.

\subsection{Learning Capabilities of Space}

In a space that transforms in response to human mood and activity, the occupant's role is anticipatory [34]. The patient may engage in various creative explorations of space with possible aesthetic goals [35]. Interaction may become playful or whimsical at times as the occupant explores the spatial possibilities. As perception is highly subjective, the occupant's personal choices and/or perceived feelings of space may also change over time. A specific environment may not be always preferable by a patient for carrying out the same activity. Recalling information over a longer time is a factor we considered. ("Spaced retrieval" (SR) is an evidence-based method for improving encoding and retrieval [36].) We are also considering the model of an "Experience Machine" [37] as a possible medical application for behavior-based methods of medical care.

The space must have the capacity to observe and "learn" from the occupant and update its data. Based upon updated data future interactions can happen. The adaptive process of the interactive living space involves neural network and "deep learning." Neural networks - that mimic how the brain functions-are trained in the same manner a child learns through examples. The learning process will be perceptionbased. Knowledge from different related disciplines will also be an integral part of learning. The space-if enabled the capacity to learn-will make spatial decisions that are personalized and customized to individual user. 
For the space to learn, the patient must have the capacity to provide subjective inputs: i.e., modify and make necessary adjustments (making space more intimate, more spacious or warmer etc.). A gesture-driven natural user interface (NUI) can be provided to the patient for providing qualitative input.

\subsection{Investigating Construction Materials}

The current practices of interactive spaces in hospitals demonstrate use of LED technology, projections and projection mapping, Virtual Reality and Augmented Reality technologies integrated with wireless sensors. Smart materials and nanotechnology open up new possibilities for the construction of the adaptive, interactive space requiring further investigation. Smart materials, such as shape memory alloys, have property changing capabilities - thermal, chemical, magnetic or electrical-when interacting with various environmental factors. In Kinect motion sensor-based interactive installations, shape memory alloy springs have been combined with skin-like tensile membrane fabric that can bend and stretch like muscles in response to motion and voice commands [38].

The programmable "Smartwrap" material-a polyester film substrate utilized in many interactive installations-can change its color, transparency, brightness and image displays in response to human activities [39]. Smart paints with embedded nanoparticles are able to change color, shape or emit sound through interaction with environmental conditions [40]. The nanotech-based interactive smart paint "WallSmart" has the capacity to change its color controlled via Bluetooth and smart phone [41]. Nanotech based smart paints or coatings also have thermal control capabilities [42]. Wide view angles are now possible with thin polymer layers used for heads-up displays: organic, fluorescent or reflective [43].

Smart glass when exposed to temperature or light changes in color, transparency and illumination [44]. Smart mirrors have in-built cameras, wi-fi and artificial intelligence that responds to voice commands, facial expressions and provides health analysis for the user [45]. Coelux is a nanotechnology-based LED window that can imitate the sunlight and the blueness of the sky [46]. It is an optical system that can simulate the warmth and coolness of sunlight, and shadow conditions that can be potentially used inside hospitals. Metamaterials are composite structures that can be programmed to respond to light in various ways [47]. It can create invisibility (cloaking) of an object by bending light in specific ways.

\section{CONCLUSION}

The importance of sensory pleasing environments, circadian rhythm-based treatments and personalization of patient's room has been acknowledged in recent healthcare design practices. Rapid advancements in LED lighting systems and interactive 'smart' technologies support variable space concepts where spaces can be personalized by selecting the color and lighting of a room based on patient's needs. The perceptual variability of space presented in this paper is guided by various types of data. For any specific type of medical condition, experiments must be conducted to gather userspecific medical and aesthetic data. Data from healthcare providers regarding a large variety of patients was obtained for future development of this project.

The concept can be practically implemented and tested with available technologies (e.g., LED systems, VR technologies or projection mapping etc.). However, further research is necessary in the field of intelligent materials that will allow for a wider range of perceptual variability of space. AaS-although a work in progress-will provide an architectural service that is sustainable to a large number of people in need of medical care. The sustainability is in relation to energy conservation, maintenance, materials etc. with blockchain guaranteeing privacy and protection of digital ownership. Very fast-changing developments in 5G technology, which also influence the IoT aspects pertinent to this project, justify small-scale experiments through which some of the assumptions made so far can be validated.

\section{REFERENCES}

[1] Larissa MacFarquhar. 2018. The Comforting Fictions of Dementia Care. (October 2018). The New Yorker. Retrieved October 23, 2018 from https://www.newyorker.com/magazine/2018/10/08/the-comfortingfictions-of-dementia-care

[2] Sue Hahn. 2012. Environments and autistic spectrum conditions. Nursing Times 108, 49 (December 2012), 23-5. www.nursingtimes.net

[3] Mihai Nadin and Asma Naz. 2018. Architecture as service: a case of design on demand (DoD). J Ambient Intell Human Comput. (December 2018) https://doi.org/10.1007/s12652-018-1147-y

[4] Mihai Nadin. 2007. Grant application, AnticipationScope and the Anticipatory Profile, 2007 NIH Director's Pioneer Award Program (DP1)

[5] Mihai Nadin. 2010. Play's the Thing. A Wager on Healthy Aging, Serious Game Design and Development. In Serious Game Design and Development: Technologies for Training and Learning, Jan Cannon Bowers and Clint Bowers (Eds.). IGI Global, USA, 150-77. DOI: 10.4018/978-1-61520-7398.ch009

[6] Mihai Nadin. 2013. Quantifying Anticipatory Characteristics. The AnticipationScope and the Anticipatory Profile. In Advanced Intelligent Computational Technologies and Decision Support Systems, Barna Iantovics and Roumen Kountchev (Eds.). Springer, New York/London/Heidelberg, 143-160

[7] Mihai Nadin. 2018. Redefining medicine from an anticipatory perspective. Progress in Biophysics and Molecular Biology 140, 21-40. https://doi.org/10.1016/i.pbiomolbio.2018.04.003

[8] Nilanjan Dey and Amira S. Ashour. 2017. Ambient Intelligence in Healthcare: A State-of-the-Art. Global Journal of Computer Science and Technology 17 (3-H)

[9] Sokratis Kartakis, Vangelis Sakkalis, Panagiotis Tourlakis, Georgios Zacharioudakis, and Constantine Stephanidis. 2012. Enhancing Health Care Delivery through Ambient Intelligence Applications. Sensors 12(9), 1143511450. https://doi.org/10.3390/s120911435

[10] Artemis Kyrkou and Fani Vavili. 2014. Innovative Materials in Children's Hospital Design. XXV International Union of Architects Congress, Durban, South Africa. DOI: 10.13140/2.1.3676.5442

[11] Morgana Matus. 2013. Interactive Woodland Wiggle Installation Encourages Kids to Play During a Hospital Stay. Retrieved March 10, 2017 from https://inhabitat.com/inhabitots/interactive-hospital-wallencourages-kids-to-play-while-they-heal/

[12] Designboom. 2013. Interactive Installation at Children's Hospital by Jason Bruges Studio. Retrieved April 12, 2017 from https://www.designboom.com/design/interactive-installation-atchildrens-hospital-by-jason-bruges/ 
[13] Bertha Coombs. 2012. Interactive Technology Soothes Kids in Emergency Rooms. CNBC. Retrieved March 10, 2017 from https://www.cnbc.com/id/46718168

[14] TUDelft. Designing Interactive Hospital Environments. Retrieved October 24, 2018 from https://www.tudelft.nl/en/ide/research/researchlabs/applied-labs/designing-interactive-hospital-environments

[15] GestureTEK Health. 2016. Interactive Environments and Entertainment Systems for Medical Facilities. Retrieved October 24, 2018 from http://www.gesturetekhealth.com/solutions/interactive-environmentsmedical-displays

[16] Francis D. K. Ching. 2007. Architecture: Form, Space, and Order. Wiley, Hoboken

[17] Peter Loewenberg. 2012. Architectural and Emotional Space. In Center 17: Space \& Psyche, Elizabeth A. Danze and Stephen M. Sonnenberg (Eds.). Center for American Architecture and Design, Canada, 16-41

[18] Gerald Franz. 2005. An Empirical Approach to the Experience of Architectural Space. Ph.D. Dissertation. Max Planck Institute for Biological Cybernetics, Tübingen and the Bauhaus University, Weimar

[19] Gaston Bachelard. 1994. The Poetics of Space. Beacon Press, Boston

[20] Peter Zumthor. 2010. Thinking Architecture. Birkhäuser, Basel

[21] Gerald Franz. 2006. Space, color, and perceived qualities of indoor environments. In Environment, Health and Sustainable Development (IAPS 19 Conference Proceedings on (D-Rom), Alexandria, Egypt

[22] Maud Hårleman. 2007. Daylight Influence on Colour Design: Empirical study on Perceived Colour and Colour Experience Indoors. Ph.D. Dissertation. Royal Institute of Technology, Sweden

[23] Adam Alter. 2012. Drunk Tank Pink. The Penguin Press, New York

[24] Stine Louring Nielsen, Carsten Friberg, and Ellen Kathrine Hansen. 2018. The Ambience Potential of Coloured Illuminations in Architecture. Ambiances 4. DOI: 10.4000/ambiances.1578

[25] Steen E. Rasmussen. 2000. Experiencing Architecture. MIT Press, Cambridge

[26] Peter Zumthor. 2006. Atmospheres: architectural environments; surrounding objects. Birkhäuser, Basel

[27] Juhani Pallasmaa. 2005. The Eyes of the Skin: Architecture and the Senses. Wiley-Academy, Chichester

[28] Asma Naz and Mihai Nadin. 2018. Application of Fuzzy Logic in Design of an Aesthetics-Based Interactive Architectural Space. IJARITAC 9(2), 113-134. DOI: 10.5958/0975-8089.2018.00012.X

[29] Asma Naz, Regis Kopper, Ryan P. McMahan, and Mihai Nadin. 2017. Emotional qualities of VR space. In 2017 IEEE Virtual Reality Conference, Los Angeles, CA, USA. 3-11. https://doi.org/10.1109/VR.2017.7892225

[30] Asma Naz. 2017. Interactive Living Space for Neo-Nomads: an Anticipatory Approach. Ph.D. Dissertation. The University of Texas at Dallas, TX, USA

[31] Quan Z. Sheng, Elhadi M. Shakshuki, and Jiangang Ma. 2014. Advances in ambient intelligence technologies. J Ambient Intell Hum Comput 5(3), 341. https://doi.org/10.1007/s12652-013-0200-0

[32] César Benavente-Peces, Andreas Ahrens, and Joaquim Filipe. 2014 Advances in technologies and techniques for ambient intelligence. $J$ Ambient Intell Human Comput 5(5), 621-622. https://doi.org/10.1007/s12652-014-0244-9

[33] Don Tapscott and Alex Tapscott. 2016. Blockchain Revolution: How the Technology Behind Bitcoin Is Changing Money, Business, and the World. Penguin Publishing Group, New York

[34] Asma Naz. 2016. Interactive living space design for neo-nomads: Anticipation through spatial articulation. In Anticipation across disciplines, Mihai Nadin (Ed.). Springer, Basel, 393-403

[35] Mihai Nadin. 2010. Anticipation and the artificial: aesthetics, ethics, and synthetic life. AI Soc. 25(1), 103-118

[36] Helen C. Kales, Laura N. Gitlin, and Constantine G. Lyketsos. 2015. Assessment and management of behavioral and psychological symptoms of dementia. BMJ 350(h369). doi: https://doi.org/10.1136/bmj.h369

[37] Robert Nozick. 1974. The Experience Machine, Anarchy, State, and Utopia. Basic Books, New York, 42-45

[38] Michael Fox. 2016. Interactive Architecture: Adaptive World (Architecture Briefs). Princeton Architectural Press, New York

[39] Robert Kronenburg. 2007. Flexible: architecture that responds to change. Laurence King, London

[40] Bogusława Konarzewska. 2017. Smart materials in architecture: useful tools with practical applications or fascinating inventions for experimental design? IOP Conf Series: Materials Science and Engineering 245(052098). https://doi.org/10.1088/1757-899X/245/5/052098

[41] Wallsmart: an interactive paint. 2014. Materia. Retrieved July 23, 2015 from https://materia.nl/article/wallsmart-interactive-paint

[42] Pravin Khandve. 2014. Nanotechnology for building material. International Journal of Basic and Applied Research 4, 146-151

[43] David L. Chandler. 2014. Seeing things: a new transparent display system could provide heads-up data. MIT News. Retrieved February 15, 2015 from http://news.mit.edu/2014/seeing-things-a-new-transparent-displaysystem-could-provide-heads-up-data-0121

[44] Orkan Telhan et al. 2010. Interaction design with building facades. In The 4th tangible and embedded interaction conference (TEI'10), Cambridge, Massachusetts, USA, 291-94

[45] Ashley Clark Thompson. 2018. The mirrors at CES had a lot to say about your life and looks. Cnet. Retrieved January 19, 2018 from https://www.cnet.com/news/tech-mirrors-atces-2018-lot-to-say-aboutyour-life-looks

[46] Robert Bain. 2015. Reviewed: CoeLux skylight. LUX. Retrieved July 20, 2015 from http://luxreview.com/review/2015/03/coelux-skylight

[47] Tomer Stav et al. 2018. Quantum entanglement of the spin and orbital angular momentum of photons using metamaterials. Science 361(6407), 1101-1104. https://doi.org/10.1126/science.aat9042 